\title{
Recent Trends in Dielectrophoretic Applications towards Medical Diagnostics
}

\section{Srivastava SK*}

Department of Chemical and Materials Engineering, University of Idaho, Moscow, USA

\section{Editorial}

There is an ever rising need in the area of medical diagnostics for early detection, high quality medical care, and new technologies that are portable and cheap, especially in the underdeveloped and developing countries. The driving force beyond this biomedical diagnostic application is the emphasis for accuracy, preventive point-of-care, and using minimally invasive samples like saliva, peripheral blood, or urine.

Recent technological advances in micro-fluidics and lab-on-achip technology has yielded in integrated medical micro-devices performing all the necessary cell manipulation steps on raw samples: cell pre-filtering, fractionation, target cell isolation, cell concentration or focusing, cell lyses, marker molecule trapping, and detection. This lab-on-a-chip technology, involving miniaturization of analytical techniques, is expected to enable highly complex laboratory tests to move from the central laboratory into non-laboratory settings. Novel electrical devices assembled by using micro-fluidics are an area of technological advancement.

There is an increasing level of interest in developing techniques for physically manipulating cells. Some of them include optical tweezers, acoustic forces, electrical forces, and surface modifications. Manipulating cells by electric forces has been the most common technique and can be classified depending on whether the electrical forces act on the particle's fixed or induced charge. Dielectrophoresis is one such electrical technique that acts on the particle's induced charge and can be created to translate, rotate, stretch and manipulate.

Dielectrophoresis (DEP), an electrokinetic effect, was discovered by Herbert A. Pohl in 1951 and later he coined the term in his book "Dielectrophoresis the behavior of neutral matter in nonuniform electric fields" in 1978. This research dates back to the early 1960's, but rapid advancement in this field started in the late 1980's. When a polarizable particle is subjected to non-uniform electric field, the force exerted on the particle causes it to move towards high or low field density regions; this behavior is known as dielectrophoresis. The motion of the particle is quantified by the polarity of charges in addition to the magnitude generated by the applied electric field and this phenomenon is often referred to as 'classical DEP'. DEP is observed only when non-uniform electric field is exerted on the cells because coulomb forces generated on both sides of the particle are different, thus facilitating motion of the particle towards regions of electric field maxima or minima. Non-uniformity could be achieved by employing metal electrodes or insulated structures embedded in the micro channel. DEP is a versatile technique that could be operated using either AC or DC electric fields or a combination of both.

DEP has been a commonly used technique for separation, trapping, and manipulation of cells, bacteria, DNA, virus, and proteins. It has also been used to monitor changes in cell states associated with activation and clonal expansion, apoptosis, necrosis, and responses to chemical and physical agents.

Recent advances to render a cheaper microfluidic option, had lead researchers to explore new fabrication techniques that include fabricating 3Dscaffolds, contactless dielectrophoresis(cDEP), 3D grapheme electrodes, etc. Extensive research using DEP has yielded in characterizing malaria-infected cells, human African Trypanosomiasis, carcinoma cells like breast cancer, leukemia, melanoma, prostate cancer, colorectal cancer, oral cancer, cervical cancer, ovarian cancer, and circulating tumor cells.

Other technological advancements have shown single cell analysis and cell fusion studies using dielectrophoresis, which can also be applied towards analyzing diseased cell interactions with healthy cells or single diseased cell analysis. Undoubtedly, dielectrophoretic microfluidic platform of disease diagnostics will generate a huge variety of new and exciting research for the betterment of human health.

The next generation cell manipulation dielectrophoretic devices would possibly make use of a number of biochemical and biophysical properties unique to diseased or infected cells that would be able to achieve high throughput and high capture rate, thus possibly detecting diseases at an early stage allowing sufficient time for diagnosis and treatment, in a much less invasive way.

${ }^{*}$ Corresponding author: Srivastava SK, Assistant Professor, Department of Chemical and Materials Engineering, University of Idaho, Moscow, USA, ID 83844, Tel: 208-885-7652; E-mail: srivastavask@uidaho.edu

Received April 28, 2015; Accepted April 30, 2015; Published May 10, 2015

Citation: Srivastava SK (2015) Recent Trends in Dielectrophoretic Applications towards Medical Diagnostics. J Biosens Bioelectron 6: e136. doi:10.4172/2155$6210.1000 \mathrm{e} 136$

Copyright: @ 2015 Srivastava SK. This is an open-access article distributed under the terms of the Creative Commons Attribution License, which permits unrestricted use, distribution, and reproduction in any medium, provided the original author and source are credited. 